\title{
Faserstruktur hartgezogener Metalldrähte ${ }^{1}$.
}

$$
\text { von }
$$

\author{
M. Ettisch, M. Polanyi und $\mathbf{K}$. Weissenberg. \\ (Mit 8 Figuren im Text.)
}

(Eingegangen am 5. 10. 21.)

Die nachfolgende Untersuchung ist die Ausarbeitung des folgenden von Becker, Herzog, Jancke, Polanyi') gefundenen eigenartigen röntgenographischen Effektes: es wurde ein harter $\mathrm{Cu}$-Draht vertikal zu seiner Längsrichtung mit monochromatischem Röntgenlicht bestrahlt und ergab dabei ein Beugungsdiagramm, das statt der bei weichen

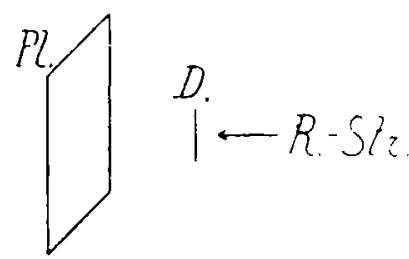

Versuchsanordnung
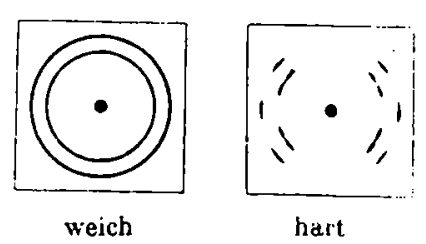

Cu-Draht.

Fig. 1.

Drähten auftretenden konzentrischen Debye-Scherrer-Kreisen Kreis bogenstreifen enthält, die in doppelt symmetrischer Anordnung den Durchstosspunkt als Mittelpunkt umgeben. (Siehe Fig. 1).

Wir fanden, dass derartige Streifendiagramme auch bei harten Drähten von $W o, F e, M o, C u, P d, A l, A g, Z n$ auftreten. Mit Hilfe der

1) Nach einem am „Deutschen Physikertag in Jena am 20. September 1921 gehaltenen Vortrag.

9) Zeitschr. f. Physik 4, 61 (1921); vgl. auch M. Ettisch, M. Polanyi, K. Weissenberg, Zeitschr. f. Physik 7 (1921). 
von Pola n y i entwickelten Methoden zur Analyse derartiger Diagramme ${ }^{1}$ ), gelang es, diese Bilder quantitativ aufzuklären. Folgender Bericht soll die rechnerischen Grundlagen nur in grossen Zügen andeuten und sich im wesentlichen auf eine Mitteilung der Ergebnisse beschränken.

Aus den bereits angedeuteten charakterischen Merkmalen obiger Streifendiagramme folgt, dass in hartgezogenen Metalldrähten die Kristallite nicht wie im weichen Draht regellos liegen, sondern in bestimmter Weise geordnet sind \%. Während nämlich im weichen Draht der ungeordneten Lage der Kristallite entsprechend in jedem Querschnitt eine unbestimmte Grosszahl verschiedener Netzebenarten liegt, enthalten alle Querschnitte des harten Drahtes nur deren wenige bestimmte. Eine solche Kristallitanordnung nennt man Faserstruktur, weil sie zuerst bei den natürlicben Fasern (Ramie, Seide) gefunden worden ist. Liegt insbesondere nur eine Netzebenenart im Querschnitt, so nennt man diese Ordnung der Kristallite einfache Faserstruktur und versteht entsprechend unter $\mathbf{z w e i f a c h e r , ~ d r e i f a c h e r}$ oder allgemeiner, mehrfacher Faserstruktur eine Ordnung, bei der 2, 3 oder allgemein wenige bestimmte Netzebenenarten im Querschnitt liegen. Von praktischer Bedeutung sind zunächst nur einfache und zweifache Faserstruktur.

\section{Metalle mit ranmzentrierten Irubisehen Gittern.}

Bei den Metallen $W o, F e, M o$, deren Elementarkörper ein raumzentrierter Würfel ist, fanden wir einfache Faserstruktur, so zwar, dass nur $\langle 110\rangle$ Flächen ${ }^{3}$ ) (Rhombendodekaederflkechen im Drahtquerschnitt liegen.

Die Fig. 2a zeigt die $\langle 110\rangle$ Ebene im raumzentrierten Elementarwürfel als Rechteck mit ausgezogenen Diagonalen angedeutet. Die Fig. 2b zeigt einen schematischen Querschnitt durch einen harten WoDraht. Die dickumränderten, unregelmässigen Felder sind Schnittflächen der einzelnen Kristallite; die Netze von Rechtecken mit ausgezogenen Diagonalen, die jedes dieser Felder überziehen, deuten an, Mitteilungen.

1) Vgl. Zeitschr. f. Phỵsik 7 (1921) sowie daselbst demnăchst erscheinende weitere

2) Eine Ordnung der Kristallite in bezug auf die Drahtachse ist bereits von Tamman, (vgl. Metallografie, 2. Auf. 1921, S. 144) mit Bestimmtheit angenommen worden auf Grund der Änderung der Leitfáligkeit und der Thermokraft beim Hartzieben von Metalldrahten.

3) Das Symbol $\langle 110\rangle$ wird als Bezeichnung einer beliebigen Rhombendodekaederfleche gebraucht, entsprechend steht (111) fur eine beliebige Ortsederfische und (100) für eine beliebige Hexaederflăche. 
dass die Netzebene, die im Querschnitt liegt, stets eine $\langle 110\rangle$ Ebene ist; aus Fig. 2a und 2b sieht man noch, dass infolge der Querlage $\operatorname{der}\langle 110\rangle$ Ebenen, alle Elementarwürfel des harten Wo-Drahtes mit zwei Flächendiagonalen ([110] Richtung) parallel zur Drahtachse stehen.

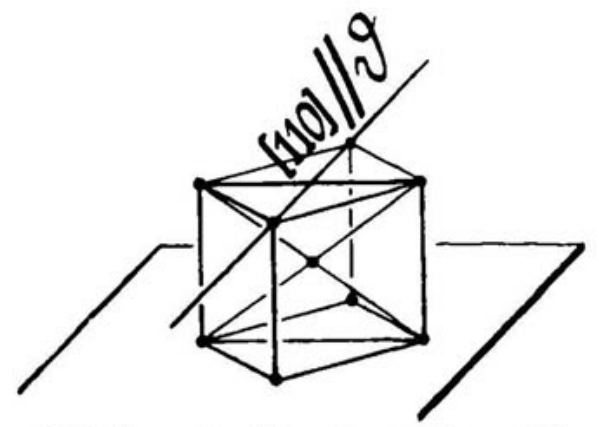

〈110〉 Ebene im Elementarwúrfel von Wo.

Fig. 2a.

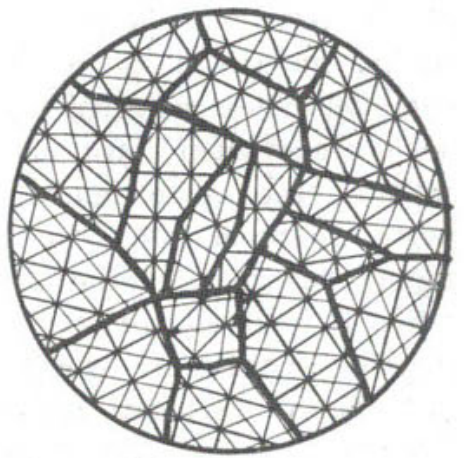

Harter Wo-Draht (Querschnitt;. $\langle 110\rangle \perp \vartheta ;[110] \| \vartheta$.

Fig. 2b.

Diese einfache Faserstruktur lässt sich röntgenographisch am leichtesten nachweisen, indem man überlegt, dass der vorhin gekennzeichnete Querschnitt im Beugungseffekt einer $\langle 110\rangle$ Kristallfläche gleichkommt,

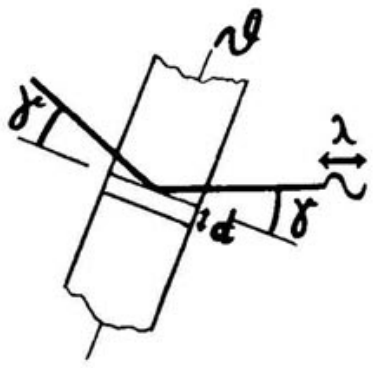

Fig. 3.

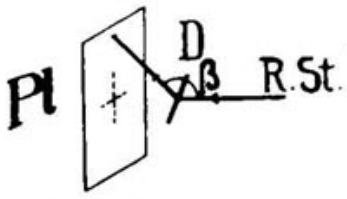

Versuchsanordnung.

Fig. 4.

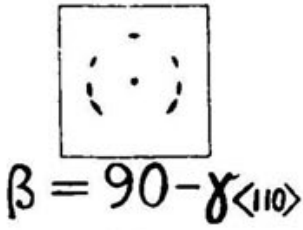

Harter $W$-Draht schief.

Fig. 5 .

d. h. einen Röntgenstrahl von bestimmter Wellenlänge nur unter einem charakteristischen Gleitwinkel $\gamma$ reflektiert ${ }^{1}$ ), wie dies die Fig. 3 veranschaulicht. Dieser ergibt sich nach Bragg zu

$$
\gamma_{\langle 110\rangle}=\arcsin \frac{\lambda}{2 d_{(110)}}
$$

1) Abgesehen von Reflexionen hoherer Ordnung. 
wobei $\lambda$ die Wellenlänge, $d_{(110)}$ den Identitätsabstand bezeichnet. Stellt man also den Drahtquerschnitt wie in Fig. 4 unter den Winkel $y_{(110)}$ zum einfallenden Strahl, so muss dann und nur dann Reflexion am Querschnitt eintreten, wenn er $\langle 110\rangle$ Ebenen enthält. Die Reflexion des Drahtquerschnittes muss sich als ein Streifen auf der vertikalen Mittellinie im Diagramm einzeichnen, da einfallender Strahl, Drahtachse und abgebeugter Strahl in einer Ebene liegen.

Fig. 5 zeigt das schematische Bild des Diagramms, das bei dieser Versuchsanordnung auftritt. Der Streifen auf der vertikalen Mittellinie beweist, dass $\langle 110\rangle$ Flächen im Drahtquerschnitt liegen. Die Deutung der anderen Streifen soll hier übergangen werden, da sie bei ihrer theoretischen Verwertung nur eine Bestätigung des obigen Befundes geben.

Man kann nun feststellen, dass der Querschnitt nicht noch andere Netzebenenarten enthält, indem man seinen Neigungswinkel zum Röntgenstrahl der Reihe nach auf die einzelnen charakteristischen Gleitwinkel der verschiedenen Netzebenen einstellt und nachweist, dass bei keiner dieser Stellungen in der vertikalen Mittellinie ein Streifen auf dem Debye-Scherrer-Kreis der betreffenden Netzebene auftritt. Doch kann man sich dieses Absuchen ersparen durch den Nachweis, dass sich schon aus der oben angenommenen Faserstruktur das vollständige Diagramm erkiăren lässt.

Indem wir die im Querschnitt liegende Netzebenenart diatrop nennen, können wir als Ergebnis der Untersuchung feststellen, dass bei den harten Metalldrăhten $\mathrm{Wo}, \mathrm{Fe}, \mathrm{Mo}$ einfache Faserstruktur vorliegt, und zwar, dass die $\langle 110\rangle$ Ebene diatrop iot; paraltel zur Drahtachse findet sich infolgedessen nur die $[110]$ Richtung vor.

\section{Metalle mit fächenzentrierten kubiseken aittern.}

Es wurde $C u, P d, A l$ untersucht. Prüft man hier den Querschnitt nach der in Fig. 4 gegebenen Anordnung auf die Netzebenenarten, die er enthält, so findet man Reflexionen des Querschnitts nur bei den Winkeln $\gamma_{(111)}$ und $\gamma_{(100)}$; infolgedessen enthält der harte Draht zwei Kristallitgruppen, von denen die eine Diatropie der $\langle 111\rangle$ Ebenen, die andere Diatropie der $\langle 100\rangle$ Ebenen aufweist. Aus dieser Kristallitanordnung lässt sich wieder das vollständige Diagramm reatlos aufklären.

Fig. 6a zeigt die Lage der $\langle 111\rangle$ Ebene und der $\langle 100\rangle$ Ebene im Elementarwürfel; Fig. $6 \mathrm{~b}$ zeigt das Schema des harten $C u$-Drahtquer- 
schnitts; entsprechend den beiden Kristallitgruppen, in denen $\langle 111\rangle, \vartheta$ bzw. $\langle 100\rangle \perp$ \& liegt, zeigt eine Gruppe von Kristallitquerschnitten das Netz der $\langle 111\rangle$ Ebene, die andere jenes der $\langle 100\rangle$ Ebene. Aus Fig. 6 a und $6 \mathrm{~b}$ erkennt man, dass wegen der Querstellung der $\langle 111\rangle$ Ebenen

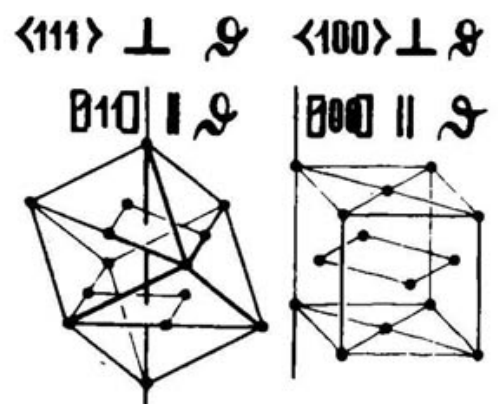

〈111) F.bone und $\langle 100\rangle$ Ebene in Elementarwúrfeln von $\mathrm{Ou}$.

Pig. 6 a.

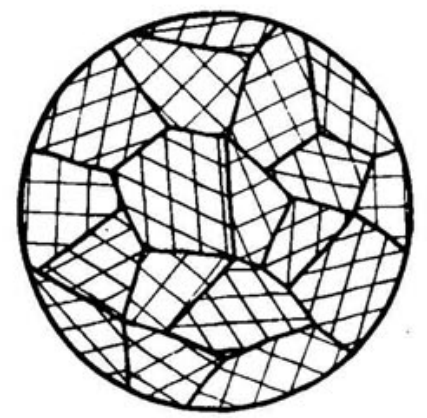

Harter Cu-Draht. (Querschnilt.)

Fig. 6b.

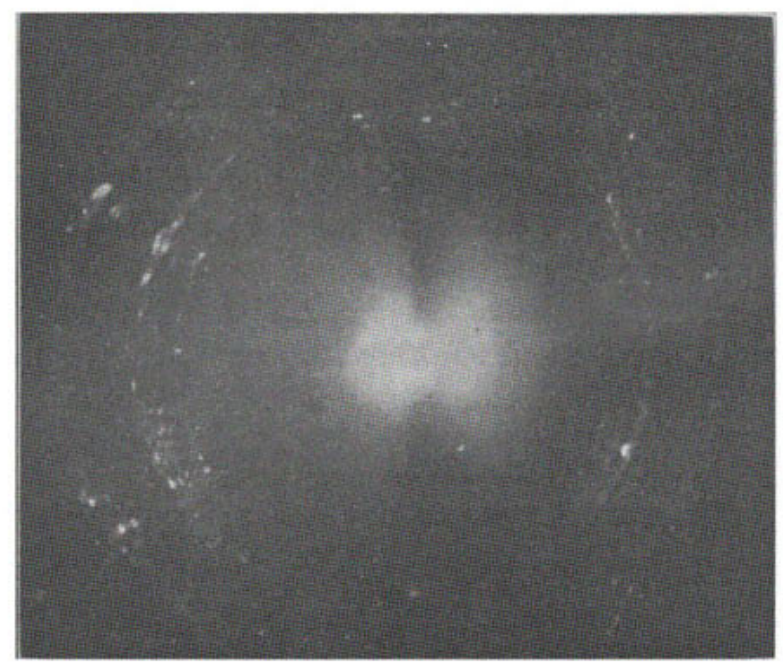

Fig. 7.

Angelassener Kupferdraht.

in der ersten Kristallitgruppe und der $\langle 100\rangle$ Ebenen in der zweiten, die Elementarkörper in der ersten mit einer Raumdiagonale (111] Richtung) und in der zweiten mit 4 Würfelkanten ([100] Richtung) parallel zur Drahtachse stehen. 
In hartgezogenen Metalldrähten von $C u, P d, A l$ besteht somit zweifache Faserstruktur, und zwar liegen die Netzebenenarten 〈111 und $\langle 100\rangle$ diatrop. Parallel zur Drahtachse finden sich infolgedessen nur die beiden kristallographischen Richtungen [111] und [100] vor.

Fig. 7 zeigt das Diagramm eines gehärteten und dann wieder angelassenen $\mathrm{Cu}$-Drahts. Die Kristallite sind durch das Anlassen merklich vergrössert und zeichnen sich als scharf begrenzte Punkte ein. Wie aus der unregelmässigen Zerstreuung dieser Punkte über die Kreise ersichtlich, ist die Faserstruktur zum grossen Teil verschwunden.

Immerhin finden sich starke Anhäufungen der Schwärzung an einzelnen Bogenstücken. Diese entsprechen dem trotz Anlassens übrig gebliebenen Teil der Faserstruktur. Die Vermessung ergibt, dass diese Schwärzungell der Diatropie der $\langle 111\rangle$ Ebene entsprechen. Beim Anlassen hat sich also die Diatropie dieser Ebene zum grossen Teil erhalten, während jene der $\langle 100\rangle$ Ebene geschwunden ist.

Frägt man danach, welche Eigenschaft einer Netzebene ihre Querstellung beim Hartziehen begünstigt, so ergibt sich im Bereiche der bisher gemachten Erfahrungen die einfache Regel, dass es die Dichte der Flächenbelegung ist. Die dichtest belegte Ebene steht immer diatrop. Daneben kann auch noch Diatropie der zweitdichtest belegten Ebene bestehen, wie bei den Metallen mit flächenzentriertem Elementarwürfel. Aus der Intensitätsverteilung kann man auch noch die Tatsache ablesen, dass im letzteren Falle die Kristallitgruppe, in der die zweitdichtest belegte Ebene diatrop ist, weniger Kristallite enthält, als die andere. Auch bei doppelter Faserung steht also die Diatropie der dichtest belegten Ebene an erster Stelle.

Die oben als Faserstruktur gekennzeichnete Anordnung der Kristallite im hartgezogenen Draht ist natürlich nicht exakt vorhanden, vielmehr stellen sich die Kristallite nur bis auf einen kleinen Streuungswinkel in die Faserordnung ein. Dieser Streuungswinkel, der somit die Grösse der Abweichung von der idealen Faserstruktur angibt, entspricht ungefähr der halben Bogenlänge der Diagrammstreifen. Aus unseren Aufnahmen lässt sich dieser Winkel auf etwa $5^{\circ}$ schätzen.

Berlin-Dahlem, Kiliser Wilhelm-Institut für Faserstoffchemie.

Leitschr. f. physik. Chemit. IC. 